\title{
BENDER E DESENHO DA FIGURA HUMANA: EVIDÊNCIA DE VALIDADE POR GRUPOS EXTREMOS
}

\section{Bender and Human Figure Drawing: evidences of validity by contrasting groups}

\author{
Fabián Javier Marín Rueda ${ }^{1}$ \\ Daniel Bartholomeu ${ }^{2}$ \\ Fermino Fernandes Sisto ${ }^{3}$
}

\section{Resumo}

O objetivo do estudo foi verificar evidência de validade para o Teste Gestáltico Visomotor de Bender em relação aos grupos extremos no Desenho da Figura Humana. Participaram 312 crianças de ambos os sexos, que cursavam de 1.a a 4. ${ }^{\text {a }}$ série do Ensino Fundamental de uma escola pública do interior do Estado de São Paulo. Os testes foram aplicados de forma coletiva em sala de aula. No caso do Bender, foram avaliadas apenas as distorções e integrações de forma nas figuras A, 1, 2, 3, 4, 5 e 7. Para o Desenho da Figura Humana, foram utilizados 49 critérios de Florence Goodenough. Os resultados evidenciaram que as crianças que apresentaram um pior desempenho no Desenho da Figura Humana apresentaram uma maior pontuação no Bender em todas as medidas estudadas. Concluiu-se que o Bender poderia ser um bom teste para avaliação do desenvolvimento cognitivo em crianças.

Palavras-chave: Bender; Desenho da figura humana; Inteligência; Instrumentos de medida.

1 Psicólogo, Mestre em Psicologia e Doutorando do Programa de Estudos Pós-Graduados em Psicologia da Universidade São Francisco. Bolsista CAPES. Rua José Marciano Filho, 9 Vila Cristo redentor, Itatiba-SP, CEP:13251-420. E-mail: marinfabian@yahoo.com.br

2 Aluno do Programa de Estudos Pós-Graduados em Psicologia da Universidade São Francisco. Bolsista CAPES.

3 Doutor pela Universidad Complutense de Madrid, Livre-docente pela Unicamp e docente do curso de Psicologia e do Programa de Estudos Pós-graduados em Psicologia, da Universidade São Francisco, Câmpus Itatiba-SP. 


\section{Abstract}

The aim of this study was to verify evidences of validity for the Bender Gestalt Visomotor Test in relation to groups should by the Human Figure Drawing. 312 male and female, attending at the $1^{\text {st }}$ to $4^{\text {th }}$ grades of public school Basic Education from the state of São Paulo have participated. The tests were collectively applied in their classroom. Concerning to the Bender, distortions and integrations of form in the figures 1, 2, 3, 4, 5 and 7 were evaluated. Florence Goodenough 49 criteria were used to assess the Human Figure Drawing. The results showed that the children with worse performances in the Human Figure Drawing greater scores in the Bender Test in all studied measures. Results suggest that the Bender could be a good test for cognitive development in children.

Keywords: Bender; Human figure drawing; Intelligence; Measure instruments.

\section{Introdução}

Inúmeros estudos indicam que o Teste de Desenho de Figura Humana e o Teste Gestáltico Visomotor de Bender estão entre os mais utilizados e mais relevantes na avaliação psicológica no Brasil (Alves, 2002; Noronha, 2002; Vendramini \& Noronha, 2002). O Teste Gestáltico Visomotor de Bender (TGB) foi desenvolvido por Lauretta Bender em 1938, tendo como referência os desenhos elaborados por Max Wertheimer para definir as leis de organização perceptual. Foram selecionadas nove figuras consideradas pela autora como as mais representativas para avaliar a ação sensório-motriz. Nesse sentido, Bender (1938) considera que a habilidade sensório-motora pode variar em razão do nível maturacional do indivíduo além de seu estado patológico funcional.

Bender (1938) utilizava os cartões com finalidade clínica, não tendo criado um sistema objetivo de correção que pudesse atribuir pontuações aos desenhos, avaliando-os apenas qualitativamente. Se, por um lado, isso dificultou a utilização do instrumento, por outro, serviu de estímulo para que diversos pesquisadores estudassem e desenvolvessem novas propostas de correção e interpretação dos desenhos, sendo alguns deles passíveis de quantificação. No que concerne aos principais sistemas de correção criados, Field, Bolton e Dana (1982) destacaram que entre as décadas 50 e 60 foram desenvolvidos pelo menos oito sistemas. No Brasil, os sistemas de correção mais utilizados são o de Clawson (1982) e o de Santucci e Pêcheux (1981).

Tendo por base o caráter evolutivo associado à aprendizagem das funções gestálticas visomotoras, Koppitz (1975) procurou distinguir, dentre as distorções ocorridas nos desenhos, aquelas relacionadas à imaturidade perceptiva e aos fatores emocionais. Assim, o sistema de correção desenvolvido parte da premissa de que o comportamento visomotor é uma habilidade e sua mensuração pode ser obtida pela reprodução de padrões com distintos níveis de complexidade.

A tais aquisições de habilidades visomotoras estão relacionadas o desenvolvimento do conceito do corpo pela criança. Assim, se determinadas condições produzirem déficits visomotores, possivelmente resultaria numa distorção do esquema corporal (Evans, 1999).

Ao lado disso, sabe-se que os Desenhos da Figura Humana (DFH) também refletem o conceito que a criança possui do corpo. Em outros termos, a criança, ao desenhar uma pessoa humana, demonstra o que conhece do corpo humano, e não somente o que vê no seu cotidiano (Goodenough, 1926; Harris, 1963).

Florence Goodenough (1926), precursora do DFH, considerava que a criança incluiria detalhes realísticos nos desenhos à medida que fosse se desenvolvendo intelectualmente. Assim, desenvolveu um sistema de correção e avaliação dos aspectos cognitivos para o desenho da figura humana.

Muito se tem utilizado o DFH na mensuração das habilidades intelectuais de crianças. Nesses termos, pode-se citar Utsugi e Ohtsuki (1955), que encontraram correlações entre o desenvolvimento intelectual e os níveis dos desenhos. Rueda, Bartholomeu e Sisto (2004) analisaram diferenças entre crianças com e sem dificuldades de aprendizagem na escrita quanto aos indicadores evolutivos dos DFH segundo os critérios de Goodenough. Os resultados mostraram que o escore do Desenho da Figura Humana diferenciou o grupo de crianças com e sem dificuldades de aprendizagem na escrita na terceira série. O mesmo não foi encontrado quanto às crianças de segunda sé- 
rie. Assim, a evidência de validade verificada foi circunscrita a apenas uma situação, colocando em dúvida a eficiência dos DFH na detecção de problemas de aprendizagem.

Por sua vez, Jing, Yuan e Liu (1999) investigaram diferenças entre QI de crianças com e sem dificuldades de aprendizagem por meio do DFH de Goodenough-Harris e do WISC. As correlações encontradas entre esses instrumentos no grupo de crianças com dificuldades de aprendizagem foi baixa. No entanto, essas crianças diferenciaram-se do grupo controle em relação a alguns detalhes nos desenhos, sugerindo a possibilidade de alguma desordem de habilidades cognitivas. Nesse sentido, Fabry, Bertinetti e Joseph (1990) administraram o DFH e o WISC-R em crianças de seis a 10 anos. Dentre os resultados, constataram que os escores do DFH correlacionaram-se mais fortemente com o QI de performance do que com o QI verbal.

Ao lado disso, Sisto (2000) investigou a validade do $\mathrm{DFH}$ de Goodenough quanto à avaliação do desenvolvimento cognitivo de acordo com as Provas Piagetianas em crianças. O Desenho da Figura Humana apresentou correlações com as três provas piagetianas de conservação de massa e comprimento e de imagem mental, o que sugere a possibilidade de encontrar padrões para avaliar a tendência do desenvolvimento cognitivo baseada nos modelos piagetianos por meio dos DFH.

Estudando pacientes com retardo mental, Upadhyaya e Sinhá (1974) aplicaram o Teste Gestáltico Visomotor de Bender (TGB) e o DFH, encontrando que sujeitos com QI mais baixo no DFH apresentaram maiores pontuações no Bender. Os autores verificaram ainda que esses dois instrumentos são freqüentemente utilizados para se obter estimativas das capacidades cognitivas dos indivíduos.

Uma breve revisão da literatura permitiu identificar algumas pesquisas que relacionassem 0 TGB com o DFH. Sob essa perspectiva, Koppitz, Sullivan, Blyth e Shelton (1959) verificaram até que ponto o TGB e o DFH administrados na primeira série preveriam a realização escolar ao final do ano, encontrando que ambos os instrumentos possuem a habilidade de predizer o desempenho acadêmico, e que o poder de predição é implementado ao serem utilizados conjuntamente.

Ainda, Bandeira e Hutz (1994) investigaram a predição do rendimento escolar na primeira série por intemédio do $\mathrm{DFH}, \mathrm{TGB}$ e Raven. Dentre os resultados, verificou-se que, apesar de todos os testes apresentarem correlações significativas com a medida de desempenho acadêmico, tomada ao final do ano letivo, somente o Bender e os itens evolutivos do DFH contribuíram na explicação da variância do rendimento escolar. Assim, o TGB e o DFH podem servir como instrumentos no diagnóstico precoce de dificuldades de aprendizagem.

Por sua vez, Rueda et al. (2005) investigaram as relações entre o TGB e o DFH em crianças do Ensino Fundamental. Os resultados indicaram correlações negativas e significativas entre o DFH e o TGB. Desse modo, concluiuse que quanto melhor o desempenho das crianças no DFH, menor a pontuação no Bender, sugerindo uma melhor maturação visomotora. Outra constatação foi que há elementos comuns entre os instrumentos, apesar de mensurarem constructos aparentemente distintos.

Tendo em vista esses aspectos considerados, denota-se a importância da maturação vis-motora no desenvolvimento conceitual avaliado pelos DFH. Assim, nessa pesquisa, será investigado mais detidamente até que ponto crianças com bom e mau desempenho no DFH são discriminados nas medidas de integração e distorção de forma avaliadas pelo TGB.

\section{Método}

\section{Participantes}

Participaram da pesquisa 177 crianças, sendo 93 (52,5\%) do sexo masculino e 84 (47,5\%) do sexo feminino, de 1. ${ }^{\text {a }}$ a $4 .^{\text {a }}$ série do Ensino Fundamental de uma escola pública do interior do Estado de São Paulo. As idades variaram entre 7 e 10 anos (média 8,62 anos e desvio-padrão de 1,15).

Para dividir os grupos extremos de crianças, separaram-se $25 \%$ de crianças com menor e maior pontuação no DFH. Dessa forma, o Grupo 1, referente aos 25\% que apresentaram menor pontuação, ficou com 45 crianças, com uma média de idade de $8,34(\mathrm{dp}=1,07)$ e o Grupo 2, referente ao $25 \%$ com maior pontuação, com 47 crianças com idade média de 8,87 $(\mathrm{dp}=1,19)$. 


\section{Instrumentos}

Teste do Desenho da Figura Humana

O Teste do Desenho da Figura Humana propõe-se a avaliar aspectos cognitivos em crianças. Neste estudo, optou-se por 49 itens a serem analisados segundo o critério de Florence Goodenough. A correção foi feita porpresença ou ausência dos itens, atribuindo-se 1 para presença e 0 para ausência, sendo que a pontuação total por sujeito foi produto da soma total das pontuações de cada item.

\section{Teste Gestáltico Visomotor de Bender}

O Teste Gestáltico Visomotor de Bender foi construído para fornecer um índice de maturação perceptomotora e consiste de nove figuras (A, 1, 2, 3, 4, $5,6,7$ e 8) para serem copiadas da melhor maneira possível sem qualquer tipo de auxílio mecânico.

Neste estudo, analisaram-se os itens referentes à distorção e integração de forma nas figuras A, 1, 2, 3, 4, 5 e 7, sendo utilizados os critérios de Koppitz. A correção foi feita atribuindo-se um ponto para cada emo cometido pela criança.

Com base na correção dos itens, foram criadas medidas, produtos da soma de itens. A distorção de forma, somatónio dos pontos atribuídos às figuras analisadas nesse quesito; a integração de forma, so- matório dos pontos atribuídos às figuras analisadas nesse quesito; e a pontuação geral, somatório das medidas anteriores.

\section{Procedimento}

Os instrumentos foram aplicados coletivamente em sala de aula. No Desenho da Figura Humana foi solicitado às crianças para desenhar uma pessoa numa folha de papel sulfite da melhor forma possível e com o máximo detalhes, informando-lhes que era permitido o uso da borracha, a qualquer momento, e que o desenho deveria ser feito a lápis.

No caso do Bender foi solicitado às crianças para copiarem os desenhos apresentados numa folha de papel sulfite da melhor forma possível, informando que era permitido o uso de borracha a qualquer momento. Entretanto, não foi permitida a utilização de qualquer ajuda mecânica.

\section{Resultados}

Para análise dos resultados, foram formados os grupos extremos de crianças em relação ao DFH. Assim, o Grupo 1 ficou formado pelas crianças que apresentaram menos de 19 pontos no teste e o Grupo 2 , com aquelas que tiveram mais de 29 pontos. No desenho da figura humana, as pontuações e freqüência dos itens do Grupo 1 são apresentados na Figura 1.

\section{Figura 1 - Freqüências das Pontuações do Grupo 1 no Desenho da Figura Humana.}

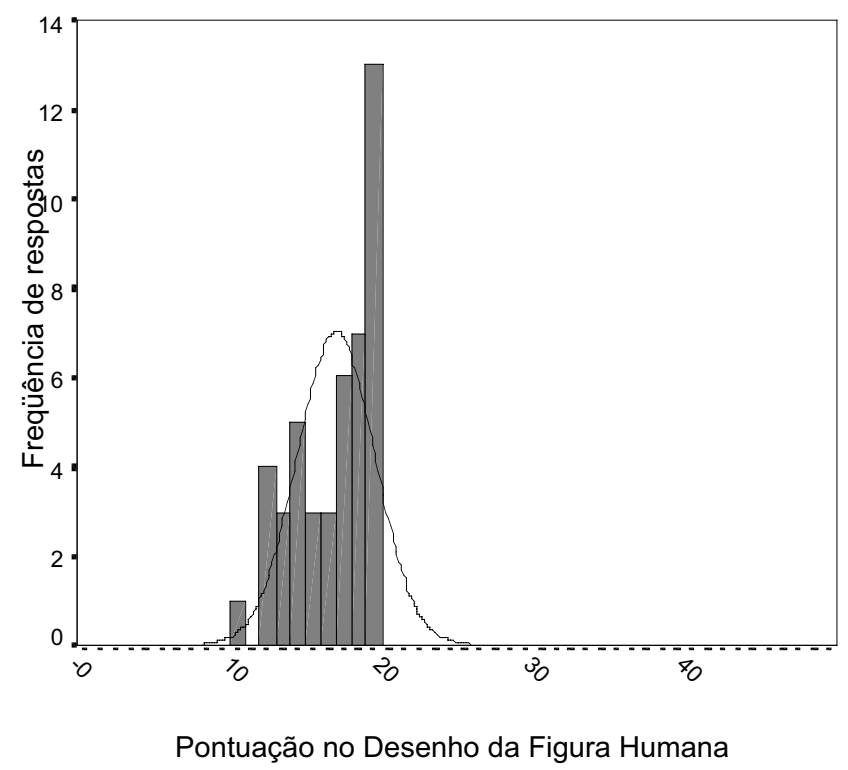


No Desenho da Figura Humana as crianças do Grupo 1 apresentaram uma média de 16,33 pontos $(\mathrm{dp}=2,59)$. A moda foi 19 itens e a mediana 17 , ou seja, $50 \%$ das crianças desse grupo desenharam menos de 17 itens e o $50 \%$ restante entre 17 e 19 itens.
Os resultados obtidos e a distribuição das freqüências dispostas na Figura 1 evidenciaram ainda uma concentração de pontos entre 17 e 19 itens (57,8\%). A porcentagem de crianças que desenharam menos de 17 itens foi de $42,2 \%$. No caso do grupo 2 no DFH, as pontuações e freqüências são apresentadas na Figura 2.

\section{Figura 2 - Freqüências das Pontuações do Grupo 2 no Desenho da Figura \\ Humana.}

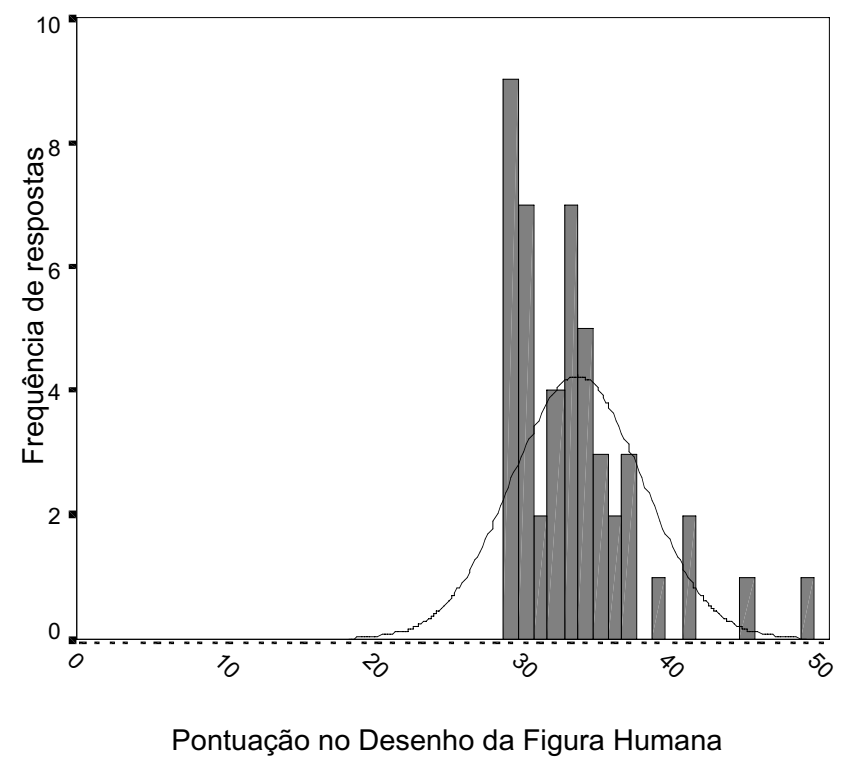

As crianças do Grupo 2 apresentaram uma média de 33,30 pontos $(\mathrm{dp}=4,33)$ no Desenho da Figura Humana. A moda foi 29 itens e a mediana 33, ou seja, $50 \%$ das crianças do grupo desenharam mais de 17 itens e o 50\% restante entre 29 e 33 itens.
Com a finalidade de verificar possíveis diferenças de média nas medidas estudadas no TGB em relação ao desempenho no DFH, foi utilizada a prova t de Student, adotando o nível de significância de 0,05 . Os resultados dessa análise encontram-se na Tabela 1. 


\section{Tabela 1 - Média, desvio-padrão, valores de t e p entre grupos ex tremos na pontuação no DFH em relação às medidas do Bender.}

\begin{tabular}{|c|c|c|c|c|c|}
\hline & Grupo & Média & $\mathrm{DP}$ & $\mathrm{t}$ & $\mathbf{p}$ \\
\hline \multirow{2}{*}{ Distorção na figura A } & 1 & 0,42 & 0,50 & \multirow[t]{2}{*}{3,328} & \multirow[t]{2}{*}{0,001} \\
\hline & 2 & 0,13 & 0,34 & & \\
\hline \multirow[t]{2}{*}{ Integração na figura $\mathrm{A}$} & 1 & 0,06 & 0,25 & \multirow[t]{2}{*}{0,054} & \multirow[t]{2}{*}{0,957} \\
\hline & 2 & 0,06 & 0,25 & & \\
\hline \multirow{2}{*}{ Distorção na figura 1} & 1 & 0,08 & 0,29 & \multirow{2}{*}{$-0,279$} & \multirow[t]{2}{*}{0,781} \\
\hline & 2 & 0,11 & 0,31 & & \\
\hline \multirow[t]{2}{*}{ Integração na figura 2} & 1 & 0,02 & 0,15 & \multirow[t]{2}{*}{1,022} & \multirow[t]{2}{*}{0,309} \\
\hline & 2 & 0,00 & 0,00 & & \\
\hline \multirow{2}{*}{ Distorção na figura 3} & 1 & 0,44 & 0,50 & \multirow{2}{*}{1,457} & \multirow{2}{*}{0,149} \\
\hline & 2 & 0,30 & 0,46 & & \\
\hline \multirow[t]{2}{*}{ Integração na figura 3} & 1 & 0,38 & 0,49 & \multirow[t]{2}{*}{1,500} & \multirow[t]{2}{*}{0,137} \\
\hline & 2 & 0,23 & 0,43 & & \\
\hline \multirow{2}{*}{ Integração na figura 4} & 1 & 0,22 & 0,42 & \multirow{2}{*}{2,625} & \multirow[t]{2}{*}{0,010} \\
\hline & 2 & 0,04 & 0,20 & & \\
\hline \multirow[t]{2}{*}{ Distorção na figura 5} & 1 & 0,44 & 0,50 & \multirow[t]{2}{*}{$-0,427$} & \multirow[t]{2}{*}{0,670} \\
\hline & 2 & 0,49 & 0,51 & & \\
\hline \multirow{2}{*}{ Integração na figura 5} & 1 & 0,02 & 0,15 & \multirow{2}{*}{$-0,973$} & \multirow[t]{2}{*}{0,333} \\
\hline & 2 & 0,06 & 0,25 & & \\
\hline \multirow[t]{2}{*}{ Distorção na figura 7} & 1 & 0,62 & 0,49 & \multirow[t]{2}{*}{6,049} & \multirow[t]{2}{*}{0,000} \\
\hline & 2 & 0,11 & 0,31 & & \\
\hline \multirow[t]{2}{*}{ Integração na figura 7} & 1 & 0,33 & 0,48 & \multirow[t]{2}{*}{3,425} & 0,001 \\
\hline & 2 & 0,06 & 0,25 & & \\
\hline Distorção de forma & 1 & 2,02 & 1,29 & 3,512 & 0,001 \\
\hline & 2 & 1,13 & 1,15 & & \\
\hline Integração de forma & 1 & 1,04 & 0,95 & 3,237 & 0,002 \\
\hline & 2 & 0,47 & 0,75 & & \\
\hline Pontuação geral & 1 & 3,07 & 1,86 & 4,231 & 0,000 \\
\hline & 2 & 1,60 & 1,45 & & \\
\hline
\end{tabular}

A Tabela 1 evidenciou que a distorção de forma nas figuras A e 7, a integração de forma nas figuras 4 e 7, a distorção e integração de forma geral e a pontuação geral do Bender apresentaram diferenças estatisticamente significativas entre os grupos extremos. Os itens integração de forma nas figuras A, 2, 3 e 5, assim como os itens de distorção de forma nas figuras 1, 3 e 5 não apresentaram diferenças estatisticamente significativas em relação aos grupos extremos estudados. Deve-se destacar que o Grupo 1 apresentou número médio de erros maior em todos os itens avaliados pelo Teste de Bender que apresentaram resultados significativos.

\section{Discussão}

O presente trabalho objetivou analisar qual a relação entre o desempenho no Teste Gestáltico Visomotor de Bender e grupos extremos na pontuação do Desenho da Figura Humana. Assim, verificou-se que sete medidas do TGB não diferenciaram tais grupos, enquanto as restantes quatro medidas, assim como também a integração de forma, distorção da forma e pontuação total apresentaram diferenças significativas nos grupos com melhor e pior desempenho no DFH. Estes achados poderiam indicar uma possível revisão da avaliação feita nas figuras do Bender, com a finalida- 
de de aprimorar os resultados finais fornecidos pelo teste, para mensurar de uma melhor forma o caráter maturacional que o instrumento se propõe a medir.

Pela análise realizada, verificou-se que as crianças que apresentaram um pior desempenho no DFH apresentaram também uma maior pontuação no TGB em todas as medidas. Assim, pode-se concluir que o Desenho da Figura Humana poderia ser um bom preditor de crianças que podem vir a apresentar algum atraso no desenvolvimento cognitivo. Nesse sentido, os achados desta pesquisa corroboram os resultados se Rueda et al. (2005), no sentido de que quanto maior a pontução no $\mathrm{DFH}$, menor a pontuação no TGB.

Ainda, esta pesquisa também confirmou os achados de Upadhyaya e Sinhá (1974), ou seja, crianças que apresentaram um pior desempenho no Teste Gerstáltico Visomotor de Bender apresentaram também uma idade mental menor no Teste do Desenho da Figura Humana. Embora estes dados não possam ser tomados como conclusivos, eles indicam uma tendência que vem sendo verificada pela literatura científica ao longo das décadas.

\section{Referências}

Alves, I. C. B. (2002). Instrumentos disponíveis no Brasil para avaliação da inteligência. In: Primi, R. (Org.). Temas em avaliação psicológica. Campinas: IBAP.

Bandeira, D. R., \& Hutz, C. S. (1994). A contribuição dos testes DFH, Bender e Raven na predição do rendimento escolar na primeira série. Psicologia: Teoria e pesquisa, 10 (1).

Bender, L. (1938). A visual motor Gestalt test and its clinical use. New York: The american Orthopsychiatric Association..

Clawson, A. (1982). Bender infantil: Manual de diagnóstico clínico. Porto Alegre: Artes Médicas.

Evans, A. L. (1999). Does deficient sensorimotor experience affect drawing of human figures? Pediatric Rehabilitation, 2(3), 37 39.
Fabry, J. J., \& Bertinetti, J. F. (1990). A construct validation study of the human figure drawing test. Perceptual and Motor Skills, 70(2), 465-466.

Field, K., Bolton, B., Dana, R. H. (1982). An evaluation of three Bender-Gestalt scoring systems as indicators of psychopathology. Journal of Clinical Psychology, 38, 838842.

Goodenough, F. L. (1926). Measurement of intelligence by drawings. New York: Harcourt, Brace \& World.

Harris, D. B. (1963). Children's drawings as measures of intellectual maturity. New York: Harcourt, Brace \& World.

Jing, J., Yuan, \& C., Liu, J. (1999). Study of human figure drawings in learning disabilities. Chinese Mental Health Journal, 13(3), 133-134.

Koppitz, E. M. (1975). The Bender-Gestalt test for young children: Research and application 1963-1973. New York: Grime \& Stratton.

Koppitz, E. M., Sullivan, J., Blyth, D., Shelton, J. (1959). Prediction of first grade school achievement with the Bender Gestalt Test and human figure drawing. Journal of Clinical Psychology, 15, 164-168.

Noronha, A. P. P. (2002). Os problemas mais graves e mais freqüentes no uso dos testes psicológicos. Psicologia: Reflexão e crítica, 15(1), 135-142.

Rueda, F. J. M., Bartholomeu, D., Sisto, F. F. (2005). Desenho da figura humana e Teste de Bender: Um estudo correlacional. Revista Psicologia Argumento, 23(39), 39-44.

Rueda, F. J. M., Bartholomeu, D., Sisto, F. F. (2004). Desenho da figura humana e aprendizagem da escrita. In: Machado, C., Almeida, L., Gonçalves, M., Ramalho, V. (Orgs.). Avaliação psicológica: Formas e contex tos. Braga: Psiquilíbrios. 
Santucci, H., \& Pêcheux, M. G.(1981). Prova gráfica de organização perceptiva para crianças de 6 a 14 anos. In: Zazzo, R. Manual para o exame psicológico da criança. São Paulo: Mestre Jou.

Sisto, F. F. (2000). Relationship of the Piagetian cognitive development to human figure drawing. Child Study Journal, 30(4), 225-232.

Upadhyaya, S., \& Sinhá, A. K. (1974). Some findings on psychodiagnostic test with young retarded adults. Indian Journal of Clinica Psychology, 1(2).
Utsugi, E., \& Ohtsuki, K. (1955). A study on the human figure drawing of children. Tohoku Psychologica Folia, 14, 131-146.

Vendramini, C. M. M., \& Noronha, A. P. P. (2002). Estudo comparativo entre testes de inteligência e de personalidade. Psicologia Reflexão e Crítica, 2(33), 413-426.

Recebido em/received in: 14/03/2006 Aprovado em/approved in: 18/10/2006 